\title{
Research into Navigation, Collaborative Interaction, and Gigapixel Displays
}

\author{
Roy A. Ruddle and David J. Duke \\ Visualization \& Virtual Reality Research Group, \\ School of Computing \\ University of Leeds \\ Leeds, UK \\ \{r.a.ruddle; d.j.duke\}@leeds.ac.uk \\ http://www.comp.leeds.ac.uk/vvr/
}

\begin{abstract}
Research by the Visualization \& Virtual Reality Research Group (School of Computing, University of Leeds, UK) includes themes that focus on navigation, collaborative interaction, and gigapixel displays. The group also carries out research into visualization techniques and systems, including new systems technologies for visualization, and tools for investigating features within large datasets. This article summarizes that research and describes current projects that are taking place: Virtual trails to aid real-world navigation, Mobile geophysics, Communication breakdown in collaborative VR, Cancer diagnosis with a VR Microscope, Visual analytic interfaces for optimization, and Overlays for graph exploration.
\end{abstract}

Keywords: Navigation; Collaborative Interaction; Gigapixel Displays; Visual Analytics; Visualization

\section{HISTORY}

Leeds was one of the first universities in the UK to buy a computer (1957), and that event was a springboard for undergraduate teaching (1966) and computing-based research. The School of Computing has been researching virtual reality for more than 20 years, initially with an emphasis on manufacturing assembly, and now with a focus on navigation, gigapixel displays and scientific visualization.

Our work has always covered both basic research and applied research, with the latter including domains such as automotive design, law, medicine and petroleum engineering. Some of the earliest research was presented at the $2^{\text {nd }}$ VRAIS symposium (now renamed the IEEE Virtual Reality Conference), and that work led directly to the establishment of a successful spin-off company (Icona Solutions; http://www.iconasolutions.com/) whose software allows the aesthetic effect of manufacturing variation (tolerance stack-up, etc.) to be visualized.

\section{OBJECTIVES}

The central theme that underpins the Visualization \& Virtual Reality Research Group's work is one of scale. We conduct research into applications where fundamental challenges are provided by the sheer scale of the data that is involved (in scientific, medical, and information visualization), and perform basic research that underpins our applied work from both front-end (navigation, interaction \& visual encoding) and back-end (visual representation; computational systems; data access) perspectives.

\section{RESEARCH THEMES}

\section{A. Navigation}

This theme both researches issues specific to navigation in VR and informs our fundamental understanding of the cognitive processes involved in human navigation in virtual and real environments. Vision is generally assumed to be the dominant source of sensory information for navigation, and when researchers highlighted how disoriented people often became in VR the cause was assumed to be the crude nature of the graphical environments being rendered [1]. However, we now know that people often remain disoriented even when virtual worlds contain rich visual detail [2]. This has led to studies conducted using an omnidirectional treadmill, which shows how translational body-based information boosts spatial learning [3] (see Figure 1). In turn, this highlights the need for further research into "walking" interfaces for the navigation of large-scale virtual worlds.

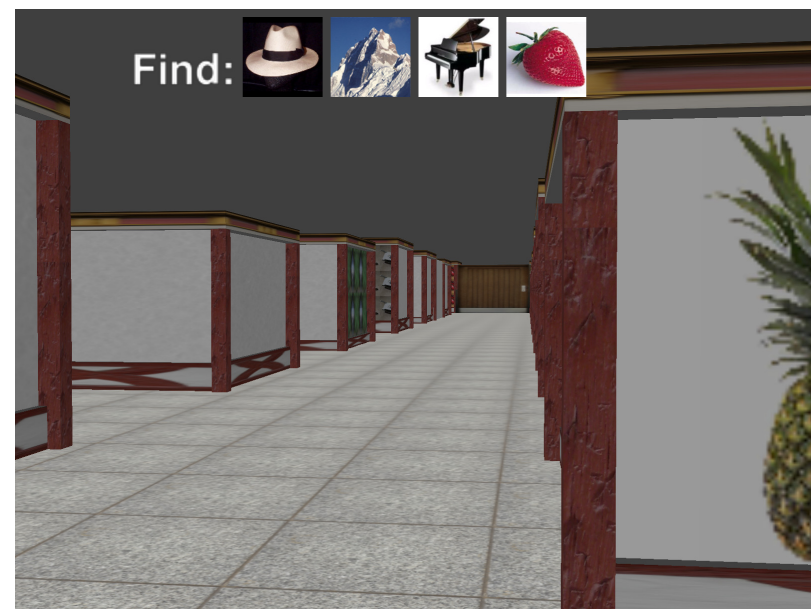

Figure 1. Navigation in a virtual marketplace [3]. 
Complementing this is research into effective ways of aiding navigation in VR, particularly through the use of trails. The challenge is to develop ways of automatically condensing a spider's web of raw movement data into trail information that is actually useful. Laboratory evaluations indicate that our trailgeneration algorithms help people navigate more effectively when they return to a virtual world after an absence [4].

\section{B. Collaborative Interaction}

One of the advantages of VR is it allows people to perform tasks that would be impossible in the real world (so-called "magic" interfaces [5]). Building on our work in navigation, we have developed a suite of techniques called mobile group dynamics that help people work together in large-scale virtual worlds and were evaluated using a town planning scenario. The research was extended to $4 \mathrm{D}$ worlds by incorporating the concept of virtual time [6] (see Figure 2), which could lead to VR-style interfaces for social networking media (e.g., Twitter).

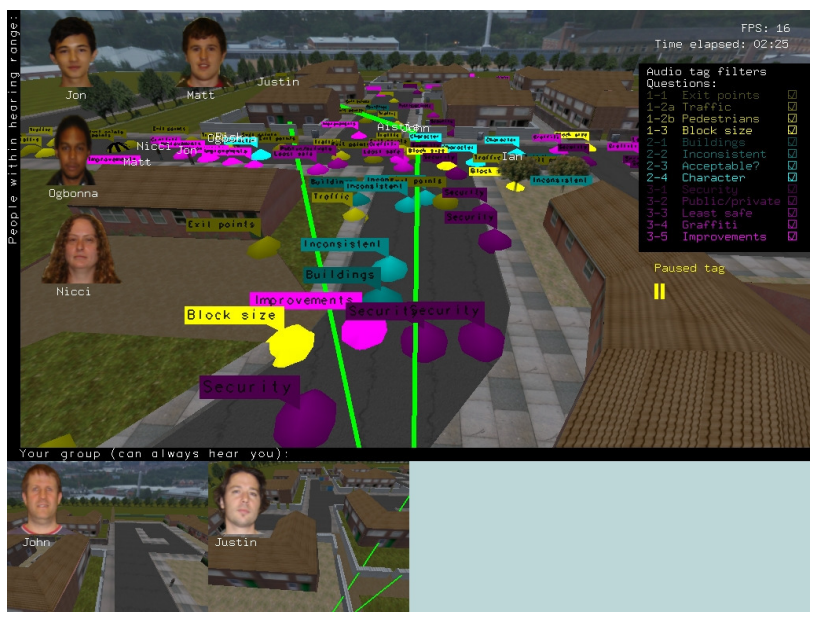

Figure 2. Virtual time in an urban planning scenario [6].

\section{Gigapixel Displays}

High-resolution wall-sized ("gigapixel") displays allow researchers to visualize extremely large amounts of data in every view. These displays are a powerful weapon for data analysis in many domains of the life and physical sciences.

Our research into the fundamentals of interaction with gigapixel displays includes the development of low-precision interaction techniques to speed up interaction [7]. Applicationbased research includes work in medical visualization [8], transport, and cancer diagnosis (see below; Figure 3).

\section{Graph Visualization}

Graphs are widely used to represent physical or logical network structures (transport \& communications; program data) along with more general relationships, e.g. links between people, events, messages, etc. However making sense of graphs is difficult. Graph layout (assignment of the nodes to positions in a $2 \mathrm{D}$ or $3 \mathrm{D}$ space) involves three-way tension between placement based on the semantics of the data (e.g., the position of a person within a social hierarchy), perceptual clarity of the display (e.g. minimizing edge crossings), and the computational cost - many graph layout algorithms are at best
$\mathrm{O}\left(|\mathrm{VI}|^{2}\right)$. For very large graphs (thousands of nodes and higher), there is insufficient screen space to discriminate fine detail, and the computational cost of processing becomes non-trivial. Our solution is twofold. First, we embed graph visualization within a high-performance pipelined visualization system. Second, we have developed a modular overlay interface that provides users with navigational cues (location of off-screen landmarks) and support for moving through the network while preserving the user's overall sense of the space [9].

\section{E. Domain-Specific Languages for Visualization}

Most systems for large scale visualization start with a lowlevel "high performance" programming language such as $\mathrm{C}++$, and then construct a series of abstractions, finally providing the user with a coarse set of components that can be assembled into an applications pipeline, via either a scripting language or a visual pipeline editor. We have recently developed a radical alternative. Functional programming provides a very high level of abstraction in which applications can be built from little "domain specific" languages specialized to particular tasks. The applications that result are often a fraction (10\%) of the size of conventional systems, simplifying the task of exploring new visual designs. More surprisingly, such programs come close to the performance of more traditional systems, while advanced tools like lazy evaluation provide a novel answer to scalability, drawing data through the pipeline strictly as needed. Our functional solution [10] to a visualization design challenge involving a terabyte of astrophysics data demonstrates the potential for this approach.

\section{CURRENT PROJECTS}

\section{A. Using Virtual Trails to Aid Real World Navigation}

Augmented reality applies VR techniques in a real-world setting. The goal of this research is to develop techniques that generate comprehensible trails from a person's everyday travels (captured by their mobile phone), to aid future navigation. For example, such an aid could guide dementia patients along routes that are chosen according to the cognitive ease of navigation, helping to reinforce knowledge of their surroundings, and potentially improve independence and quality of life.

\section{B. Mobile Geophysics}

Petroleum and mining companies routinely combine data from remote sensing and field survey work during exploration for new resources. The goal of this research is to improve the speed and quality of field surveys, via the augmented reality delivery of 3D models of survey data to the mobile devices of an exploration team. This research is in collaboration with the Centre for Integrated Petroleum and Engineering Geoscience (CiPEG) at Leeds (http://cipeg.leeds.ac.uk).

\section{Preventing Communication Breakdowns}

One of the problems of collaborative working, and collaborative VR in particular, is misunderstandings of the intentions, actions and current focus of one's partners. This results in communication breakdowns that impede the work concerned. The goal of this research is to develop types of interface that support users when they collaborate while using 
displays that have markedly different capabilities (e.g., gigapixel $\rightarrow$ desktop $\rightarrow$ mobile).

\section{Cancer Diagnosis}

Histopathology is the medical specialty concerned with diagnosing cancer and other diseases by examining tissue. Histopathologists make these diagnoses using microscopes, but it is now possible to scan the tissue to create virtual slides. However, the resulting images are extremely large (typically $100,000 \times 80,000$ pixels). In collaboration with the Leeds Institute for Molecular Medicine (LIMM) (http://www.virtualpathology.leeds.ac.uk) and the Leeds Teaching Hospitals NHS Trust, we are applying VR techniques to develop a virtual microscope that allows histopathologists to diagnose cancer quicker than, but as accurately as, with a conventional microscope. The wall-sized version of our microscope was originally developed in 2006 and renders these gigapixel images in real time [11] (see Figure 3), and a workstation-based solution is being developed and evaluated for day-to-day diagnostic work with practicing histopathologists.

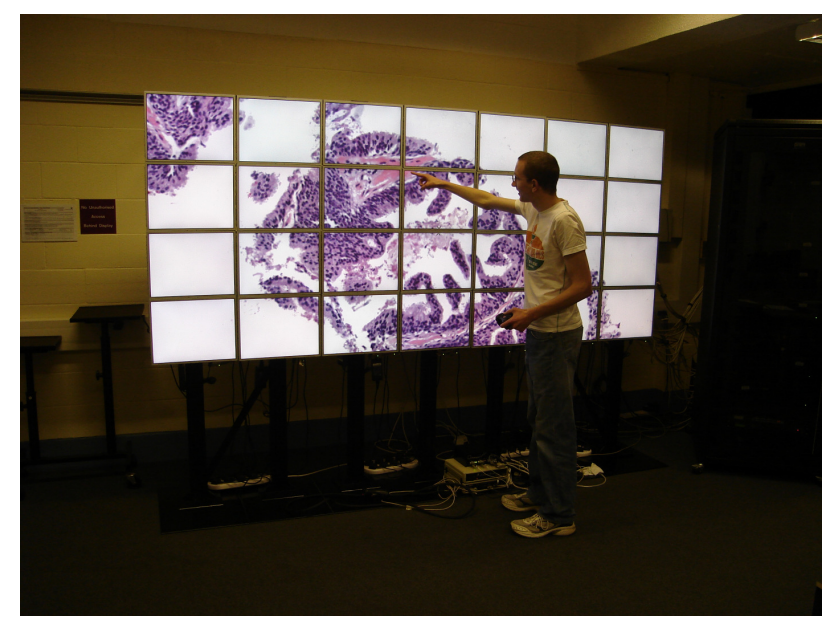

Figure 3. Use of a "gigapixel" display for a VR Microscope [11].

\section{E. Visual Analytics}

Visual analytics systems aim to transform data into knowledge by integrating human-in-the-loop visualization with the processing power of automated analysis algorithms. The purpose of visualization in the analytic process shifts from being the medium though which the user discovers knowledge itself (as is the case in conventional visualization algorithms), to being the medium that helps the user design and optimize the analysis algorithms. This change of emphasis is essential because only automated algorithms are capable of handling the quantity of data that occurs in visual analytic applications (e.g., genomics, telecommunications, security, and business transactions). Our research focuses on a new paradigm for visual analytic interfaces, which helps human experts optimize algorithms for automated processing and is currently being applied to biomedical applications [12].

\section{F. Visualizing Multicore Program Performance}

Pure functional programming languages, for example Haskell, should make it easier to exploit the parallelism provided by modern multi-core processors. However, the runtime behavior of pure functional programs is very different from that of conventional systems. Lazy evaluation, where data are only evaluated when needed to produce output, means that the locus of execution follows a complex trajectory through the program. Also compilation of a functional program through an intermediate abstract machine makes it difficult to relate low level machine instructions back to high level program abstractions. Within a project funded by Microsoft Research, we are investigating how visualization of data from the Haskell run-time system can be extended to allow programmers to finetune performance on multi-core processors. Initial work [13] has explored how to provide users with information about the location of run-time events within the program source code.

\section{CONCLUSIONS}

"Scale" is the overriding theme that binds together all of the Visualization \& Virtual Reality Research Group's research. As well our VR and human-computer interaction (HCI) research, we also study information visualization, computational topology \& geometry, and volume visualization (the latter themes led by $\mathrm{Dr}$ Hamish Carr). Potential students and collaborators are welcome to contact the Group's staff about the following themes:

- Roy Ruddle: Navigation, Collaborative interaction, Gigapixel displays, Real-time interaction, Medical visualization, Genomics, Geophysics, VR, HCI..

- David Duke: Graph visualization, DomainSpecific Languages for Visualization, Functional programming, Visual abstractions, Information visualization, Bioinformatics, HCI.

- Hamish Carr: Computational topology, Computational geometry, Volume visualization, Visualization algorithms, Abstraction \& synthesis, Medical visualization, Geophysics.

\section{REFERENCES}

[1] R. A. Ruddle, S. J. Payne, and D. M. Jones, Navigating buildings in "desk-top" virtual environments: Experimental investigations using extended navigational experience", Journal of Experimental Psychology: Applied, vol. 3: pp. 143-159, 1997.

[2] R. A. Ruddle and S. Lessels, "For efficient navigational search humans require full physical movement but not a rich visual scene", Psychological Science, vol. 17, pp. 460-465, 2006.

[3] R. A. Ruddle, E. Volkova, and H. H. Bülthoff, "Walking improves your cognitive map in environments that are large-scale and large in extent", ACM Transactions on Computer-Human Interaction, vol. 18(2), Article $10,2011$.

[4] R. A. Ruddle, "Generating trails automatically, to aid navigation when you revisit an environment", Presence: Teleoperators and Virtual Environments, vol. 17, pp. 562-574, 2008.

[5] Bowman, D. A., E. Kruijff, J. J. LaViola, and I. Poupyrev. "3D user interfaces: Theory and practice", London: Addison-Wesley, 2004.

[6] T. J. Dodds, and R. A. Ruddle, "Using mobile group dynamics and virtual time to improve teamwork in large-scale collaborative virtual environments", Computers \& Graphics, vol. 33, pp. 130-138, 2009. 
[7] C. Rooney, and R. A. Ruddle, "Improving window manipulation and content interaction on high resolution, wall-sized displays", International Journal of Human-Computer Interaction, in press.

[8] C. E. Goodyer, J. H. Hodrien, J. D. Woof, P. Kohl, and K. W. Brodlie, "Using high resolution displays for high resolution cardiac data. Philosophical Transactions of the Royal Society Part A; Mathematical, Physical and Engineering Sciences”, vol. 367, pp. 2667-2677, 2009.

[9] C. Myers, and D. Duke, "A map of the heap: Revealing design abstractions in runtime structures", In Proceedings of the ACM Symposium on Software Visualization (SOFTVIS), pp. 63-72, 2010.

[10] D. J. Duke, R. Borgo, C. Runciman, and M. Wallace, "Huge data but small programs: Visualization design via multiple embedded DSLs",
Lecture Notes in Computer Science, vol. 5418, pp. 31-45, Berlin: Springer Verlag, 2009

[11] D. Treanor, N. Jordan Owers, J. Hodrien, P. Quirke, and R. A. Ruddle, "Virtual reality Powerwall versus conventional microscope for viewing pathology slides: an experimental comparison", Histopathology, vol. 5, pp. 294-300, 2009.

[12] A. J. Pretorius, M.-A. Bray, A. E. Carpenter, and R. A. Ruddle, "Visualization of parameter space for image analysis", IEEE Transactions on Visualization and Computer Graphics, in press.

[13] Wortmann, P., "Weaving Source Code into Threadscope", Haskell Implementors' Workshop, Tokyo, 2011. 